\title{
Los retos de la medicina personalizada
}

\author{
Luis Torre-Bouscoulet* ${ }^{*}$ \\ *Instituto Nacional de Enfermedades Respiratorias Ismael Cosío Villegas, \\ Ciudad de México. \\ Trabajo recibido: 12-XII-2015; aceptado: 14-XII-2015
}

A pesar de que en la Facultad de Medicina nos decían "existen enfermos, no enfermedades», el ejercicio de la medicina ha estado basado por muchos años en el diagnóstico nosológico; es decir, en las enfermedades. Dicho paradigma es práctico porque facilita la toma de decisiones terapéuticas, aliviando con ello la preocupación de saber lo que debemos prescribir a un paciente que manifiesta determinados síntomas. Las características de los síntomas, junto con los hallazgos del examen físico y de los estudios auxiliares de diagnóstico, nos permiten concluir, por ejemplo, que un sujeto fumador durante 50 años con disnea, sibilancias, tos y expectoración, con patrón espirométrico de obstrucción grave y tomografía con enfisema centrolobulillar es portador de enfermedad pulmonar obstructiva crónica (EPOC). Con esos datos o algunos otros, clasificamos al paciente como miembro de un grupo dado (EPOC GOLD I-IV o GOLD A-D) y eso nos lleva, bajo el argumento de la "evidencia», a prescribir un tratamiento "estándar». Ejemplos semejantes podríamos mencionar en casos de asma, neumonía o cáncer.

Las Guías de Práctica Clínica (GPC), tan populares ahora, son un refuerzo a esa medicina de las «enfermedades» y no de los «enfermos». En ellas encontramos recomendaciones basadas en «evidencias» acerca del tratamiento que deben recibir los pacientes que cumplen ciertos criterios de clasificación para cierta enfermedad. El paradigma de la medicina, aquél en el que la implementación de las GPC parecía ser un avance sustancial, ha cambiado ostensiblemente. En los últimos años nos hemos adentrado poco a poco en la «medicina de las $4 \mathrm{P}$ »; la que es preventiva, predictiva, personalizada y participativa. Dichos atributos, en especial el de ser «personalizada» o «de precisión», exigen un cambio en el abordaje clínico de los pacientes, del basado en «enfermedades» al basado en «enfermos». Hoy, los enfermos tendrán características especiales que los subagrupan, aun cuando la enfermedad reciba el mismo nombre. Así, habrá asmáticos Th2 y asmáticos no-Th2 de acuerdo con su tipo de respuesta inflamatoria, o pacientes con EPOC con o sin eosinofilia periférica, o pacientes con cáncer pulmonar con mutaciones específicas que los hacen tributarios a tratamientos específicos. Las «enfermedades» parecerían ser en realidad "síndromes» en los que cada paciente con asma, EPOC, cáncer o fibrosis debe ser sometido a estudios minuciosos, no sólo clínicos o bioquímicos, sino también moleculares o genéticos, para poder saber cuál es «su» tratamiento y no «el» tratamiento. La farmacogenómica es precisamente esa disciplina científica que nos ayuda a precisar si nuestro paciente asmático, o cualquier otro, se beneficiará de un tratamiento al igual que otro con el mismo diagnóstico. Esta «personalización» de la medicina hace que los nuevos diagnósticos nosológicos sean aquellos basados en análisis moleculares o genéticos.

El modelo actual de personalización de la medicina se basa en el genoma, microbioma, metabolómica, perfil nutricional, protéomica, así como en la actividad física y el estilo de vida. Todos estos factores, en su interacción simultánea natural, contribuyen al perfil molecular personal que nos otorgará una cierta susceptibilidad a las enfermedades y cierta respuesta a las intervenciones terapéuticas. En este contexto tan individualizado imaginemos nuestro sistema de salud. Si el abordaje basado en diagnósticos sindromáticos no ha funcionado; ¿cómo podremos, en las instituciones públicas de salud, adentrarnos en el mundo de la medicina personalizada? Si por ahora los centros de salud son afortunados en tener algunos medicamentos, ¿qué será cuando debamos diagnosticar a nuestro paciente con EPOC o asma, no sólo con espirometría -que ya sería un gran avance-, sino también con mediciones moleculares o genéticas a fin de poder prescribirle el mejor tratamiento disponible para sus características «personales»"? El cáncer de pulmón ha sido una bue- 
na experiencia en esto de la medicina personalizada. La evidencia actual ha demostrado la conveniencia de «personalizar» el tratamiento en pacientes con cáncer de pulmón; sin embargo, en otras enfermedades, la personalización de la medicina podría no ser una alternativa útil en términos de la salud pública. Dejar de fumar, por ejemplo, debería ser una recomendación universal, independientemente de si el sujeto tiene características moleculares o genéticas de «resistencia» a los daños por el tabaco. Aunque exista «resistencia» para desarrollar EPOC, difícilmente la resistencia será compartida para cáncer o fibrosis o enfermedad coronaria, etcétera.

En el campo de la investigación, la medicina personalizada complica la ejecución de proyectos clínicos. Los criterios de inclusión deben tomar en cuenta las particularidades moleculares o genéticas de los pacientes para poder garantizar la validez externa de los resultados. Los resultados serán extrapolables a un grupo de pacientes que compartan las mismas «huellas» moleculares. Dichos estudios serán más costosos que los tradicionales debido a que el escrutinio de los pacientes implicará un análisis molecular detallado o a que será necesario incluir a muchos sujetos a fin de poder controlar los factores confusores.

Lo preventivo y lo predictivo del paradigma actual no son atributos nuevos. Conocemos bien las ventajas de prevenir enfermedades a partir de la habilidad predictiva de modelos matemáticos. Por ejemplo, sabemos que si pudiéramos eliminar por completo el consumo de tabaco, podríamos prevenir $85 \%$ de los casos de cáncer de pulmón y que si tratamos a los pacientes con apnea del sueño mediante equipos de presión positiva, podríamos disminuir el riesgo de enfermedades cardiovasculares en la población general; ejemplos como éstos abundan. El aspecto participativo podría estar bien representado por la adherencia de los pacientes a los tratamientos crónicos. Es bien conocido que, en el mejor de los casos aun en países desarrollados, la adherencia tera- péutica a largo plazo no supera $30-35 \%$. Sin embargo, el aspecto participativo al que se refiere el paradigma actual es más ambicioso. El sistema vertical de atención médica en el que las indicaciones eran señaladas por el médico es obsoleto. El modelo actual es horizontal, un equipo coordinado por el médico, pero en el cual intervienen otros protagonistas como el rehabilitador, el fisioterapeuta, el educador en salud, el promotor de salud, el informático, todo esto inmerso en el aspecto «participativo» del paciente. El paciente deja de ser un ente pasivo para convertirse en protagonista del proceso de atención médica. La participación del paciente es activa, no basta tomar o inhalar sus medicamentos, sino que la educación sobre su enfermedad permitirá activamente realizar procedimientos en beneficio de él mismo. Así, por ejemplo, el paciente con asma activamente medirá su función pulmonar o su fracción exhalada de óxido nítrico, y en consecuencia, él mismo -después de un proceso educativo-, habrá de decidir si debe ajustar su tratamiento o no. En algunos casos será necesario transferir la información a una enfermera respiratoria que, mediante una entrevista no presencial, le podrá hacer recomendaciones terapéuticas. Remitir cada vez más pacientes a los cada vez más saturados centros de salud u hospitales de segundo o tercer nivel es claramente un modelo insostenible. Debemos implementar estrategias eficientes de atención médica que forzosamente deben incluir la participación de los pacientes, los sistemas horizontales y la telemedicina.

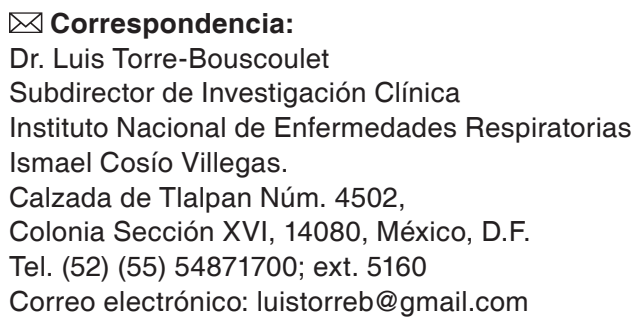

El autor declara no tener conflicto de intereses. 